\title{
R Aquarii and Gamma Ray Astronomy
}

\author{
Kenji Tanabe *† \\ Okayama Univ.Sc., \\ E-mail: tanabe@big.ous.ac.jp \\ Yuko Motizuki \\ RIKEN Nishina Center for Accelerator-based Science \\ E-mail: motizuki@riken.jp...
}

In the Antarctic ice core there exist many nitrate ion concentration spikes due to gamma ray. A part of the ice core extracted from the Japanese Antarctic station named Dome Fuji,corresponding with 11th century, shows three set of spikes.The first two possibly coincide with SN1006 and SN1054.The third one is probably due to the symbiotic binary R Auarii's outbursts (1073 and 1074AD)that were recorded in the ancient Korean documents as a "guest star". Moreover,there exist several nitrate ion spikes corresponding with other centuries. Some of the spikes without any historical records are possibly due to the gamma-ray from invisible or missing $\mathrm{SNe}$ or other (classical novae or symbiotic star )outbursts.

The Golden Age of Cataclysmic Variables and Related Objects IV

11-16 September, 2017

Palermo, Italy

\footnotetext{
* Speaker.

$\dagger$ another affiliation :RIKEN Nishina Center for Accelerator-based Science; kenjitanabe@ riken.jp
} 


\section{Introduction}

The relationship between Antarctic ice core records and supernova remnants was first pointed by Rood et al. (1979 Nature). However,such a idea is not yet successful because of lacking definite results due to their age determination,contamination from volcano explosion, no ice core records on the historical $\mathrm{SNe}$ and so on.

On the other hand there exist some ten historical records concerning the so-called Guest Stars in Korean ancient documents. Among them documents are known that describes the precise position of these guest stars on the Eastern Asian star charts. Especially a guest star in 1073 and 1074AD is thought to be identical with the Symbiotic star R Aquarii , which was pointed out by Hon-Jing Yang et al. (2005).This record can be a clue to solve the relation between the ice core records and historical star outburst.

\section{Korean historical records of $\mathbf{R}$ Aquarii}

A well-known symbiotic binary $\mathrm{R}$ Aquarii is one of the most enigmatic variable star.According to Hong -Jin Yang (2005), outburst record of this star in 1073/1074AD is described in the ancient Korean documents,including its precise position in the old Asian star atlas and brightness expression.

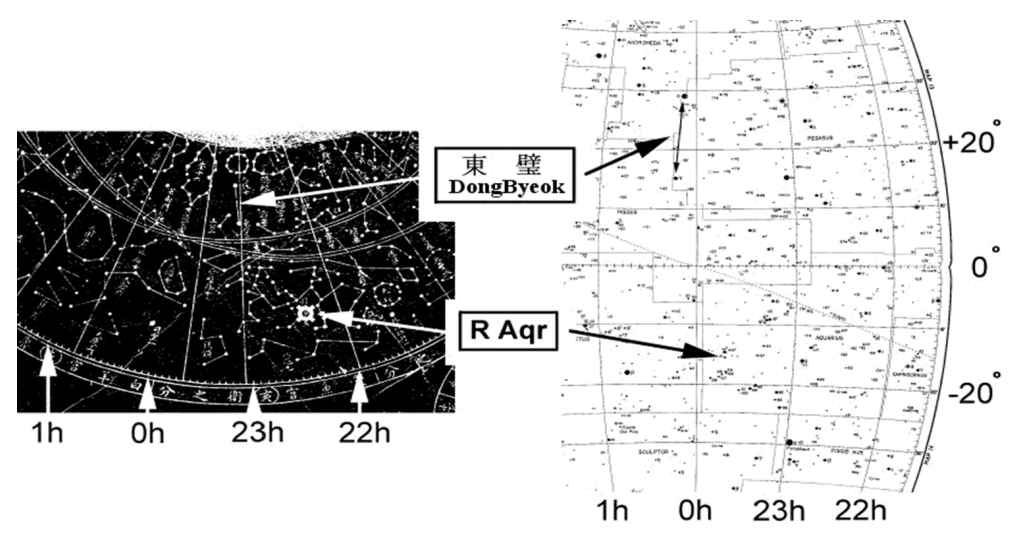

Figure 1: The position of R Aquarii both in the Korean chart and modern one.Adopted from Yang,H$\mathrm{J},(2005)$

This historical record seems to be coincident with Antarctic ice core records of 11th century extracted from the Japanese Dome Fuji station(Motizuki,Y.(2009)).At first, the third nitrate ion concentration with double peaked spike were not concerned with any historical record or supernova remnant.However we considered the possibility of not SN but a symbiotic binary R Aqr (Tanabe,K. and Motizuki,Y.(2012)).

In addition there exists a description on this brightness that the outburst was extremely bright (like “ orange” (Hon-Jing Yang (2005)).

\section{Ice Core Records in Antarctica}

As is mentioned, the relationship between Antarctic ice core records and SN remnants was 
first pointed by Rood et al.(1979).They pointed out the coincidence of the age of ice core records with the historical supernovae since 12th century(including SN1181). However their results were uncertain for their age determination and inconsistent with the results from other ice core records on supernova remnants .

On the other hand 11th Century records in the Japanese Antarctic station named Dome FUJI records are much more clear because of no record of big volcano explosion(Fig.2). Spikes coincide with 1006,1054 SNe and the third one with double peak spikes coincides with not any SN but symbiotic binary R Aquarii's 1073/1074AD outbursts recorded in the ancient Korean documents(Yang,H-.J.(2005)). As for the age determination of ice core extracted from the Dome Fuji(at the mountain top of $3800 \mathrm{~m}$ height), time-resolution(relative) $\simeq 0.5$ year,and absolute determination, using the solar activity,is of some 50 year(Fig.3) .

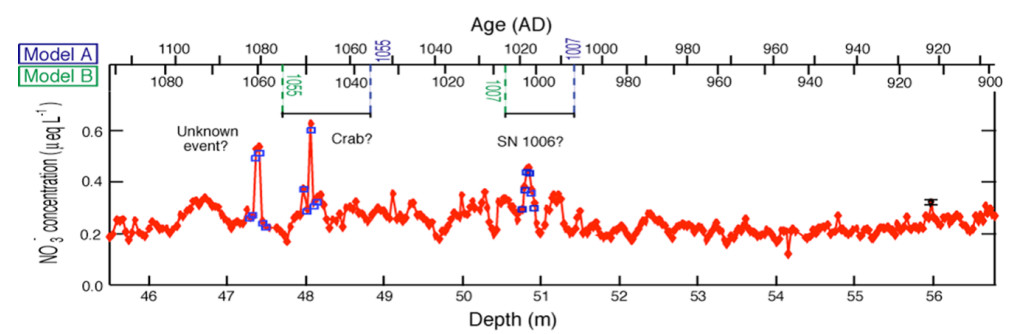

Figure 2: Dome Fuji station ice core records in 11th century.Adopted from Motizuki et al,(2009)

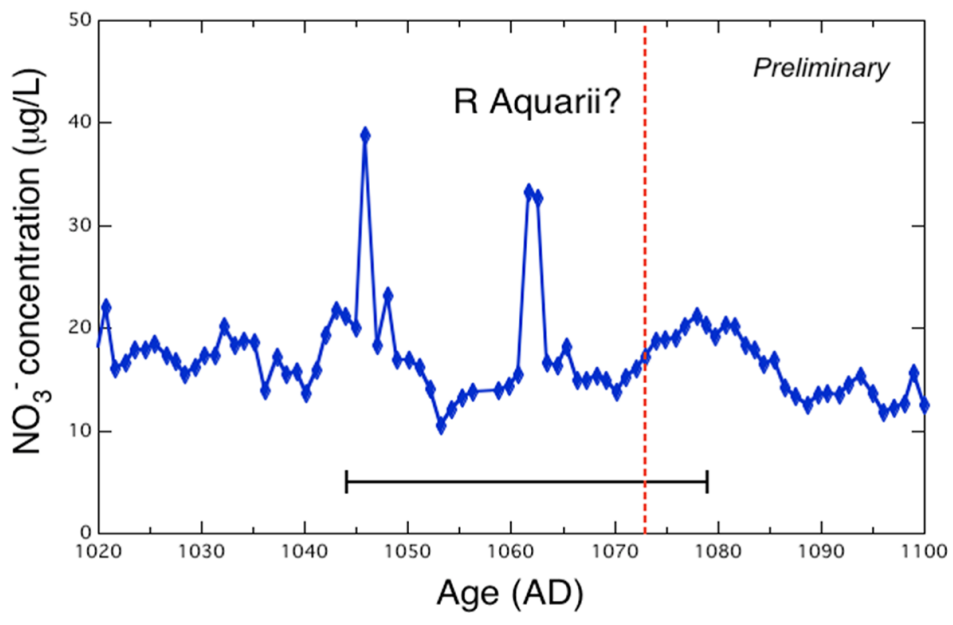

Figure 3: Enlarged picture of Figure 2.

\section{Gamma Ray Astronomy and Symbiotic Stars}

Gamma ray from from outside of the earth reacts the earth atmosphere,nitrogen,and produce nitrate ion, falling and accumulated at the top of Antarctic mountain. For the gamma ray with energy higher than $10 \mathrm{KeV}$, but lower than $0.511 \mathrm{MeV}$,Compton scattering is dominated. According to the famous Klein-Nishina formula(Klein,O. and Nishina,Y.(1929)), the cross section of the 
Compton scattering is very small. As a result gamma ray from $\mathrm{SNe}$ and $\mathrm{CNe}$ can arrive at the earth without losing energy,even from the Galactic center.In this case no optical counterpart cannot be visible but $\mathrm{SN}$ remnant can be seen afterwards.Moreover ice core can record the invisible or missing $\mathrm{SNe}$ and related objects.In fact, the distribution of gamma ray from GRB arrive quite isotropically,as is seen in the results of famous BATSE(Burst and Transient Source Experiment).

\section{Other ice core records from Dome Fuji}

An example of other ice core record from Dome Fuji station is given in Figure 4.This sample is of depth from $8 \mathrm{~m}$ to $23 \mathrm{~m}$. This corresponds with the age of probably 1600AD - 1900AD.There exist several conspicuous spikes.However solar activity and volcano explosion are possible source of spikes during this period.

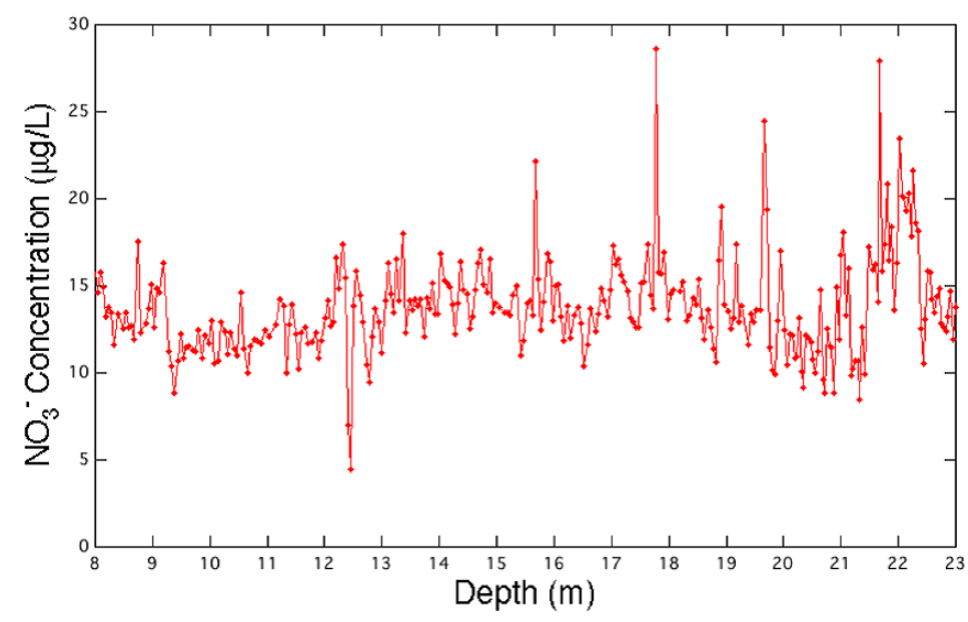

Figure 4: Ice core records from17th to 19th century.

\section{Concluding Remarks}

1. Ice core records can be the relic of the past gamma-ray from both $\mathrm{SNe}$ and probably $\mathrm{CNe}$. Spike height (nitrate ion concentration degree)can reflect gamma-ray flux(not photon energy itself).

2. Spikes can be $\gamma$-ray of invisible novae even from Galactic center $((l, b) \simeq(0,0))$.

3. The case of Classical Novae, nova-remnants disappear rapidly, except a few case including R Aqr (probably 1 event /century or less, but more frequent than the Galactic SNe).

4. 11th century age-depth relation can be a measure to determine the age of spikes. 


\section{References}

[1] Rood,R.T. et al. 1979 Nature 282,701

[2] Yang,H.-J. et al.2005 A\& A 435,207

[3] Motizuki,et al. 2009 eprint arXv0902.3446

[4] Tanabe,K. Motizuki,Y.2012 Momorie della Societa astronomia Italiana Vol.83 N.2,p.840

[5] Klein,O. and Nishina,Y. 1929 Zs.f Phys.52

\section{DISCUSSION}

JOANNA MIKOLAJEWSKA's Comment: R Aqr has well-determined distance,orbital period and binary components are resolved(see Schmid +2017 , and references herein).

KENJI TANABE: Thank you so much for your important information. (The paper is Schmid,H.M. et al. 2017 A\&A 602,A53) 